\title{
Medium-induced gluon radiation off massive quarks fills the dead cone
}

\author{
Néstor Armesto, Carlos A. Salgado, and Urs Achim Wiedemann \\ Theory Division, CERN, CH-1211 Geneva 23, Switzerland
}

(Received 8 December 2003; published 1 June 2004)

\begin{abstract}
We calculate the transverse momentum dependence of the medium-induced gluon energy distribution radiated off massive quarks in spatially extended QCD matter. In the absence of a medium, the distribution shows a characteristic mass-dependent depletion of the gluon radiation for angles $\theta<m / E$, the so-called dead cone effect. Medium modifications of this spectrum are calculated as a function of quark mass $m$, initial quark energy $E$, in-medium path length and density. Generically, medium-induced gluon radiation is found to fill the dead cone, but it is reduced at large gluon energies compared to the radiation off light quarks. We quantify the resulting mass dependence for momentum-averaged quantities (gluon energy distribution and average parton energy loss), compare it to simple approximation schemes and discuss its observable consequences for nucleusnucleus collisions at the BNL RHIC and CERN LHC. In particular, our analysis does not favor the complete disappearance of energy loss effects from leading open charm spectra at the RHIC.
\end{abstract}

DOI: $10.1103 /$ PhysRevD.69.114003

PACS number(s): 12.38.Mh, 24.85.+p

\section{INTRODUCTION}

Hadronic jets accompanying heavy quarks $c, b$ differ from light quark and gluon initiated jets. These differences can be attributed to the suppression of gluon bremsstrahlung from massive charges at small angles $\theta<m / E$, the dead cone effect [1]. Observable consequences of this mass dependence of the partonic fragmentation pattern include the softening of the light hadron spectra accompanying heavy quark jets [2], and the significant hardening of the leading charmed [3] or $b$-flavored [4] hadron. Mass-dependent dead cone conditions are implemented in the modified leading logarithmic approximation which accounts for jet multiplicity distributions [5]. They are also implemented in modern Monte Carlo simulations [6] which provide a probabilistic implementation of the perturbative part of the parton fragmentation process in the vacuum.

How is this parton fragmentation modified if the produced high-energy parent quark propagates through dense QCD matter, as is the case in ultrarelativistic nucleus-nucleus collisions at the BNL Relativistic Heavy Ion Collider (RHIC) and at the CERN Large Hadron Collider (LHC)? As a first step toward addressing this question [7], several groups [8-12] calculated to leading order in energy and for an arbitrary number of medium-induced momentum exchanges the modifications to the $q \rightarrow q g$ splitting process for energetic light quarks. These calculations indicate that medium effects can result in a significant additional energy degradation of the leading hadron which grows approximately linear with the density of the medium and approximately quadratic with the in-medium path length. Recent measurements [13-18] of high $-p_{\perp}$ hadroproduction and its centrality dependence in $\mathrm{Au}+\mathrm{Au}$ collisions at $\sqrt{s_{\mathrm{NN}}}=200 \mathrm{GeV}$ provide the first evidence [19] for this medium-induced parton energy loss. For light quarks and gluons, the formalism was also extended to the angular dependence of the medium-modified gluon radiation [20-23]. This allows discussion of medium modifications of jet shape and jet multiplicity observables [24].

For massive quarks, the formalism of medium-induced gluon radiation is not developed to the same extent. Dok- shitzer and Kharzeev [25] suggested that the dead cone effect also reduces the medium-induced gluon radiation, thus resulting in a smaller suppression of leading charmed and $b$-flavored hadrons. They estimated this effect by multiplying the medium-induced gluon spectrum for massless quarks with a transverse momentum averaged suppression factor given in Eq. (4.11) below. However, the combination of vacuum-induced and medium-induced gluon energy distributions is known to differ significantly from a simple superposition [20] due to the non-trivial interplay of interference effects and elastic scattering. Hence, it is conceivable that the medium-induced part of the gluon radiation differs significantly from this averaged dead cone approximation. Going beyond this approximation may also be needed to distinguish mass-dependent final state effects from non-linear modifications of the initial gluon distribution for which open charm production may be a sensitive probe [26-29].

This motivates one to parallel for the massive case the calculations of medium-induced gluon radiation that exist for the massless case. To this end, two groups [30-33] presented detailed calculations of parton energy loss for massive quarks. However, these calculations are limited to the average energy loss of massive quarks only. Here, we go beyond these studies (i) by providing the first analysis of the double differential medium-induced gluon distribution as a function of transverse momentum and gluon energy and (ii) by comparing this gluon distribution to the massless limit for a wide parameter range in quark mass, in-medium path length and medium density. In Sec. II we set up the path-integral formalism for parton energy loss. In Secs. III and IV, we summarize the results of our numerical calculations and provide simple analytical arguments for how the mass dependence shows up in the medium-induced gluon radiation. The main results and their relevance for heavy quark production in nucleus-nucleus collisions at the RHIC and LHC are discussed in the Conclusions.

\section{MEDIUM-INDUCED GLUON RADIATION OFF MASSIVE QUARKS: FORMALISM}

In this section, we introduce the path-integral formalism for medium-induced gluon radiation and briefly discuss its 


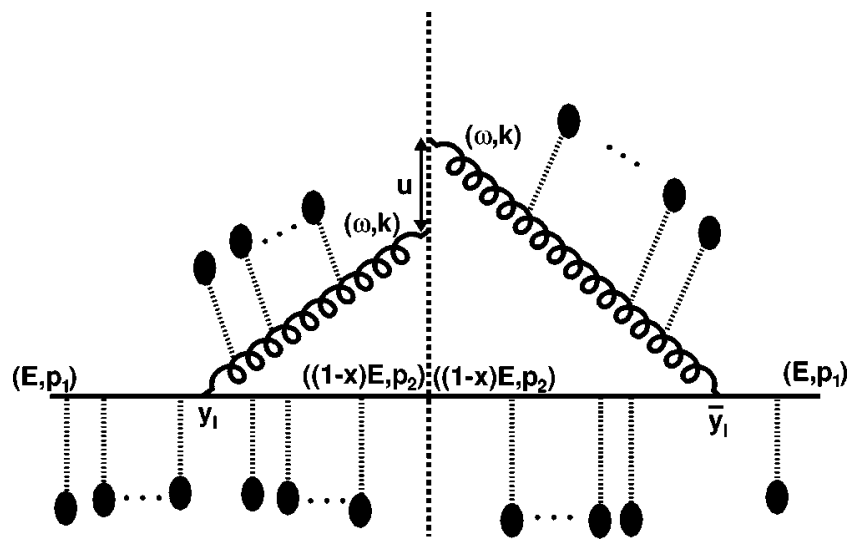

FIG. 1. A typical example of the class of multiple-scattering diagrams which are resummed in Eq. (2.1). The dashed line shows the position of the cut. The transverse coordinate $\mathbf{u}$ is conjugate to the gluon transverse momentum $\mathbf{k}_{\perp}$, and the coordinates $y_{l}, \bar{y}_{l}$ are longitudinal positions used in time-ordered perturbation theory. Further details are given in the text.

derivation, physical content and theoretical uncertainties. For a massive quark traversing spatially extended QCD matter, we show, based on Ref. [10], that the final result for the medium-induced gluon distribution can be written as

$$
\begin{aligned}
\omega \frac{d I}{d \omega d \mathbf{k}_{\perp}}= & \frac{\alpha_{s} C_{F}}{(2 \pi)^{2} \omega^{2}} 2 \operatorname{Re} \int_{0}^{\infty} d y_{l} \int_{y_{l}}^{\infty} d \bar{y}_{l} e^{i \bar{q}\left(y_{l}-\bar{y}_{l}\right)} \\
& \times \int d \mathbf{u} e^{-i \mathbf{k}_{\perp} \cdot \mathbf{u}} \exp -\left(\frac{1}{2} \int_{\bar{y}_{l}}^{\infty} d \xi n(\xi) \sigma(\mathbf{u})\right) \\
& \times \frac{\partial}{\partial \mathbf{y}} \cdot \frac{\partial}{\partial \mathbf{u}} \int_{\mathbf{y}=0=\mathbf{r}\left(y_{l}\right)}^{\mathbf{u}=\mathbf{r}\left(\bar{y}_{l}\right)} \mathcal{\mathbf { r }} \\
& \times \exp \left[i \int_{y_{l}}^{\bar{y}_{l}} d \xi \frac{\omega}{2}\left(\dot{\mathbf{r}}^{2}-\frac{n(\xi) \sigma(\mathbf{r})}{i \omega}\right)\right]
\end{aligned}
$$

Here, $\omega$ and $\mathbf{k}_{\perp}$ denote the energy and transverse momentum of the emitted gluon, respectively. The Casimir factor $C_{F}$ $=\frac{4}{3}$ determines the coupling strength of this gluon to the massive quark. For numerical results, we fix the coupling constant to $\alpha_{s}=1 / 3$ unless stated otherwise. The properties of the medium enter Eq. (2.1) in terms of the product of the time-dependent density $n(\xi)$ of scattering centers times the strength of a single elastic scattering $\sigma(\mathbf{r})$. This dipole cross section $\sigma(\mathbf{r})$ is given in terms of the elastic high-energy cross section $|a(\mathbf{q})|^{2}$ of a single scatterer in the color octet representation,

$$
\sigma(\mathbf{r})=2 \int \frac{d \mathbf{q}}{(2 \pi)^{2}}|a(\mathbf{q})|^{2}\left(1-e^{i \mathbf{q} \cdot \mathbf{r}}\right) .
$$

\section{A. Derivation of Eq. (2.1)}

We have derived Eq. (2.1) according to the techniques of Ref. [10] by resumming the multiple scattering Feynman diagrams shown in Fig. 1 to leading order in $1 / E$ in the norm and next-to-leading in the phase. Equation (2.1) describes the nuclear enhanced medium modification of the gluon radiation to leading order $\mathcal{O}\left(\alpha_{s} T\right)$. Here the dimensionless parameter $T=\int_{0}^{L} d \xi n(\xi) \sigma(\mathbf{r}=0)$ measures the opacity of the medium of length $L$. The two-dimensional transverse coordinates $\mathbf{u}, \mathbf{y}$ and $\mathbf{r}$ emerge in the derivation of Eq. (2.1) as distances between the positions of projectile components in the amplitude and complex conjugate amplitude. The longitudinal coordinates $y_{l}, \bar{y}_{l}$ integrate over the ordered longitudinal gluon emission points in amplitude and complex conjugate amplitude, which emerge in time-ordered perturbation theory (see Fig. 1). For longitudinal positions $\xi>\bar{y}_{l}$ for which a gluon is emitted in both amplitude and complex conjugate amplitude, the dipole cross section $\sigma(\mathbf{u})$ in Eq. (2.1) measures the impact parameter difference $\mathbf{u}$ between the two gluons. By Fourier transformation, this difference $\mathbf{u}$ is related to the final transverse momentum $\mathbf{k}_{\perp}$ of the emitted gluon. For longitudinal positions $y_{l}<\xi<\bar{y}_{l}$ for which a gluon is emitted in the amplitude but not yet in the complex conjugate amplitude, the dipole cross section $\sigma(\mathbf{r})$ in Eq. (2.1) measures the impact parameter difference $\mathbf{r}$ between the emitted gluon and the position of the emitting quarkantiquark system in amplitude and complex conjugate amplitude. Technically, this results again in the cross section for a dipole with two color octet legs. Physically, this reflects the fact that in a configuration space picture, the strength of the medium-induced radiation is determined by the transverse spatial displacement of the radiating charge. In the following discussion, this space-time interpretation of the internal integration variables in Eq. (2.1) plays no role. An explicit derivation and more detailed discussion of Eq. (2.1) can be found in Ref. [10].

The only mass dependence of the gluon distribution (2.1) comes from the phase factor $\exp \left[i \bar{q}\left(y_{l}-\bar{y}_{l}\right)\right]$, where $\bar{q}$ is defined as the difference between the total three-momentum of the initial quark $\left(p_{1}\right)$, and the final quark $\left(p_{2}\right)$ and gluon $(k)$,

$$
\bar{q}=p_{1}-p_{2}-k \simeq \frac{x^{2} m^{2}}{2 \omega}, \quad x=\frac{\omega}{E},
$$

with $E$ the energy of the initial quark. For the Abelian case, the same phase factor is known to give the mass dependence of medium-induced photon radiation to leading order in $x$ $\ll 1$ (see Refs. [34,35]). Paralleling the derivation of Ref. [10] for massive quarks, we have checked explicitly that this phase is the only mass dependence of the gluon distribution (2.1).

The derivation of Eq. (2.1) employs the high-energy approximation in which the medium acts as a collection of static scattering centers and the initial parton loses a small additional medium-induced amount of its total energy, $\Delta E$ $\ll E$. Thus, the result $(2.1)$ is unreliable for parameter values $\Delta E \sim \mathcal{O}(E)$. We shall try to quantify the corresponding uncertainties at the end of Secs. III and IV.

In the absence of medium effects, the gluon energy distribution (2.1) reduces to the unperturbed radiation $I^{\mathrm{vac}}$ associated with the production of a massive quark in the vacuum. 
We write the full radiation spectrum as the sum of this vacuum component and its medium modification $I^{\mathrm{med}}$,

$$
\omega \frac{d I}{d \omega d \mathbf{k}_{\perp}}=\omega \frac{d I^{\mathrm{vac}}}{d \omega d \mathbf{k}_{\perp}}+\omega \frac{d I^{\mathrm{med}}}{d \omega d \mathbf{k}_{\perp}} .
$$

By construction, both the full gluon distribution $\omega d I / d \omega d \mathbf{k}_{\perp}$ as well as the vacuum component $\omega d I^{\mathrm{vac}} / d \omega d \mathbf{k}_{\perp}$ have to be positive for all values of $k_{\perp}$ and $\omega$. In contrast, the mediuminduced modification $\omega d I^{\mathrm{med}} / d \omega d \mathbf{k}_{\perp}$ can be negative in some part of phase space: negative values correspond to a medium-induced depletion of the vacuum component.

\section{B. Approximation schemes for the path integral in Eq. (2.1)}

For realistic dipole cross sections $\sigma(\mathbf{r})$, the path integral in Eq. (2.1) is not known. Here, we shortly review the two approximation schemes in which it can be calculated. We limit the discussion to the case of a static medium with inmedium path length $L$ for which

$$
n(\xi)=n_{0} \Theta(L-\xi) .
$$

From the analysis of Ref. [36], we expect that the case of an expanding medium can be reformulated in terms of a static medium (2.5) with suitably adjusted density $n_{0}$.

\section{Opacity expansion}

The opacity expansion amounts to expanding the integrand of Eq. (2.1) in powers of $[n(\xi) \sigma(\mathbf{r})]^{N}$. Details are given in Appendix A and in Refs. [10,11]. This is a systematic expansion: resummation of all order $N$ reproduces the full result (2.1). The zeroth order in opacity, $N=0$, is the only medium-independent term and hence defines the vacuum contribution in Eq. (2.4). The first order, $N=1$, determines the leading medium dependence of the gluon radiation. For an on-shell parton scattering on a medium, the $N$ $=1$ term defines the totally incoherent radiation limit for a single scattering center times the effective number of scattering centers involved. Higher orders $N>1$ then characterize corrections due to coherence effects. For an off-shell parton, already the $N=1$ term contains interference effects between vacuum and medium-induced radiation. In this case, numerical studies [11] suggest that the contribution from $N>1$ is small. Since the $N=1$ opacity approximation can be viewed as involving a single scattering center (times an effective opacity-dependent factor), and since it tends to emphasize (semi)hard momentum transfers from the medium, it is sometimes referred to as "single hard scattering approximation."

\section{Dipole approximation}

Alternatively, one can calculate the gluon distribution (2.1) in the saddle point approximation using $\sigma(\mathbf{r}) \propto \mathbf{r}^{2}$ $[34,37]$. This so-called dipole approximation is studied in Sec. IV. Physically, the quadratic approximation $\sigma(\mathbf{r}) \propto \mathbf{r}^{2}$ implies that the perturbative high transverse momentum tails of single scattering centers are ignored. The projectile performs an exact Brownian motion in transverse phase space, as expected as a consequence of many soft momentum transfers. That is why it is sometimes referred to as "multiple soft scattering approximation."

We emphasize that both the single hard and the multiple soft scattering approximation are limiting cases of the same expression (2.1). They make substantially different assumptions about the main medium effect which stems either from many soft or from one effective hard scattering center. The realistic case can be expected to lie in between these extreme limits, and deviations between both limiting cases allow to estimate uncertainties of the approximation [23]. Remarkably, however, both approximations are known to lead to physically equivalent results for the gluon radiation off a massless parton [23]. We anticipate that the present work allows to extend this conclusion to the mass-dependent case.

\section{OPACITY EXPANSION}

In this section, we study the gluon distribution (2.1) in the opacity expansion. This expansion was motivated in Sec. II B above. Further technical details can be found in Appendix A and in Refs. [10,11].

\section{A. $N=0$ vacuum term: The dead cone effect}

In the absence of medium effects, $n(\xi)=0$, the gluon distribution (2.1) reduces to the zeroth order in opacity,

$$
\omega \frac{d I^{\mathrm{vac}}}{d \omega d \mathbf{k}_{\perp}} \equiv \omega \frac{d I(N=0)}{d \omega d \mathbf{k}_{\perp}}=\frac{\alpha_{s} C_{F}}{\pi^{2}} H\left(\mathbf{k}_{\perp}\right),
$$

where $H\left(\mathbf{k}_{\perp}\right)$ denotes the radiation term associated with the hard parton production,

$$
H\left(\mathbf{k}_{\perp}\right)=\frac{\mathbf{k}_{\perp}^{2}}{\left(\mathbf{k}_{\perp}^{2}+x^{2} m^{2}\right)^{2}} .
$$

By construction, this is the vacuum term in Eq. (2.4). It shows the dead cone effect: gluon radiation is suppressed for gluons which are emitted under small angles

$$
\frac{\mathbf{k}_{\perp}^{2}}{\omega^{2}}<\frac{m^{2}}{E^{2}} .
$$

The vacuum term (3.2) for the massive case vanishes for $k_{\perp} \rightarrow 0$, while the corresponding massless limit formally diverges like $1 / k_{\perp}^{2}$.

\section{B. $N=1$ opacity correction to the dead cone effect}

We consider a medium of spatially extended QCD matter, modeled as a collection of colored Yukawa-type scattering centers (A4) with Debye screening mass $\mu$. To first order $N=1$ in opacity, the average momentum transfer from the medium to the hard quark is $\mu$.

\section{Notation}

It is convenient to express gluon energy, transverse momentum and quark mass in rescaled dimensionless quanti- 
ties. The corresponding scaling variables are motivated by the following qualitative considerations: A gluon of energy $\omega$ decoheres from the wave function of a massless quark if its typical formation length $\bar{l}_{\mathrm{coh}}=2 \omega / \mu^{2}$ is smaller than the typical distance $L$ between the production point of the parton and the position of the scatterer. Hence, gluon radiation occurs if the phase

$$
\bar{\gamma}=\frac{L}{\bar{l}_{\mathrm{coh}}} \equiv \frac{\bar{\omega}_{c}}{\omega}
$$

exceeds unity. We thus present the energy dependence of the radiated gluon distribution in units of the characteristic gluon energy

$$
\bar{\omega}_{c} \equiv \frac{1}{2} \mu^{2} L
$$

The corresponding transverse momentum dependent phase accumulated due to scattering of the gluon is

$$
\frac{\mathbf{k}_{\perp}^{2}}{2 \omega} L=\frac{\bar{\kappa}^{2}}{\omega / \bar{\omega}_{c}}>1
$$

This makes it convenient to use the dimensionless rescaled transverse momentum variable

$$
\bar{\kappa}^{2} \equiv \frac{\mathbf{k}_{\perp}^{2}}{\mu^{2}} .
$$

We also use a rescaled quark mass. The vacuum radiation term (3.2) can be obtained by multiplying its massless limit with the dead cone factor

$$
F(\bar{\kappa}, \bar{M})=\left(\frac{\bar{\kappa}^{2}}{\bar{\kappa}^{2}+\bar{M}^{2}}\right)^{2}=\left(\frac{\mathbf{k}_{\perp}^{2}}{\mathbf{k}_{\perp}^{2}+x^{2} m^{2}}\right)^{2} .
$$

This motivates the definition of the rescaled dimensionless mass parameter

$$
\bar{M}^{2} \equiv \frac{x^{2} m^{2}}{\mu^{2}}=\frac{1}{2}\left(\frac{m}{E}\right)^{2} \frac{\bar{R}}{\bar{\gamma}^{2}}, \quad \bar{R}=\bar{\omega}_{c} L .
$$

\section{Analytical results}

We have calculated the medium modification of the gluon energy distribution (2.4) to first order in opacity (see Appendix A). In terms of the dimensionless variables introduced above, we find

$$
\begin{aligned}
\omega \frac{d I(N=1)}{d \omega d \bar{\kappa}^{2}}= & \frac{\alpha_{s} C_{F}}{\pi}\left(2 n_{0} L\right) \int_{0}^{\infty} d \bar{q}^{2} \\
& \times \frac{\left(\bar{q}^{2}+\bar{M}^{2}\right)-(1 / \bar{\gamma}) \sin \left[\bar{\gamma}\left(\bar{q}^{2}+\bar{M}^{2}\right)\right]}{\left(\bar{q}^{2}+\bar{M}^{2}\right)^{2}} \\
& \times \frac{\bar{q}^{2}}{\bar{q}^{2}+\bar{M}^{2}} \\
& \times \frac{\left(\bar{\kappa}^{2}+\bar{M}^{2}\right)+\left(\bar{\kappa}^{2}-\bar{M}^{2}\right)\left(\bar{\kappa}^{2}-\bar{q}^{2}\right)}{\left(\bar{\kappa}^{2}+\bar{M}^{2}\right)\left[\left(1+\bar{\kappa}^{2}+\bar{q}^{2}\right)^{2}-4 \bar{\kappa}^{2} \bar{q}^{2}\right]^{3 / 2}} .
\end{aligned}
$$

In the massless limit, our result reduces to the $N=1$ opacity result given in Refs. [10,36],

$$
\begin{aligned}
\lim _{m \rightarrow 0} \omega \frac{d I(N=1)}{d \omega d \bar{\kappa}^{2}}= & \frac{\alpha_{s} C_{F}}{\pi}\left(2 n_{0} L\right) \int_{0}^{\infty} d \bar{q}^{2} \\
& \times \frac{\bar{q}^{2}-(1 / \bar{\gamma}) \sin \left(\bar{\gamma} \bar{q}^{2}\right)}{\bar{q}^{4}} \\
& \times \frac{\left(1+\bar{\kappa}^{2}-\bar{q}^{2}\right)}{\left[\left(1+\bar{\kappa}^{2}+\bar{q}^{2}\right)^{2}-4 \bar{\kappa}^{2} \bar{q}^{2}\right]^{3 / 2}} .
\end{aligned}
$$

In the following subsections, we compare the full massdependent gluon distribution (3.10) to the massless limit. We also test the conjecture that this mass dependence can be described approximately by multiplying the massless expression (3.11) with the dead cone factor (3.8),

$$
\omega \frac{d I_{\text {dead }}(N=1)}{d \omega d \bar{\kappa}^{2}}=F(\bar{\kappa}, \bar{M}) \lim _{m \rightarrow 0} \omega \frac{d I(N=1)}{d \omega d \bar{\kappa}^{2}} .
$$

For finite in-medium path length $L$, Eq. (3.10) interpolates between the totally coherent and totally incoherent limiting cases of the gluon radiation spectrum. In particular, the incoherent limit is an independent superposition of three distinct radiation terms,

$$
\begin{aligned}
\lim _{L \rightarrow \infty} \omega & \left.\frac{d I(N=1)}{d \omega d \mathbf{k}_{\perp}}\right|_{n_{0} L=\mathrm{const}} \\
= & \frac{\alpha_{s} C_{F}}{\pi^{2}}\left(n_{0} L\right) \int \frac{d \mathbf{q}}{(2 \pi)^{2}}|a(\mathbf{q})|^{2}\left[-H\left(\mathbf{k}_{\perp}\right)\right. \\
& \left.+H\left(\mathbf{k}_{\perp}+\mathbf{q}\right)+R\left(\mathbf{k}_{\perp}, \mathbf{q}\right)\right] .
\end{aligned}
$$

Here, the hard radiation term (3.2) $H\left(\mathbf{k}_{\perp}\right)$ is multiplied by the probability that the hard parton interacts with the medium; the minus sign ensures probability conservation by reducing the corresponding weight of the $N=0$ vacuum contribution (3.1). For a scattering center far away from the point of quark production, interaction with the medium thus 
gives rise to two processes. (i) The vacuum radiation term $H\left(\mathbf{k}_{\perp}\right)$ is shifted to $H\left(\mathbf{k}_{\perp}+\mathbf{q}\right)$ due to medium-induced elastic scattering by a transverse momentum q. (ii) Additional gluons are produced due to bremsstrahlung off the far distant scattering center. This is described by the Gunion-Bertsch radiation term for massive quarks,

$$
\begin{aligned}
R\left(\mathbf{k}_{\perp}, \mathbf{q}\right)= & \frac{\left(\mathbf{k}_{\perp}+\mathbf{q}\right)^{2}}{\left[\left(\mathbf{k}_{\perp}+\mathbf{q}\right)^{2}+x^{2} m^{2}\right]^{2}} \\
& -\frac{2 \mathbf{k}_{\perp} \cdot\left(\mathbf{k}_{\perp}+\mathbf{q}\right)}{\left[\left(\mathbf{k}_{\perp}+\mathbf{q}\right)^{2}+x^{2} m^{2}\right]\left[\mathbf{k}_{\perp}^{2}+x^{2} m^{2}\right]} \\
& +\frac{\mathbf{k}_{\perp}^{2}}{\left[\mathbf{k}_{\perp}^{2}+x^{2} m^{2}\right]^{2}} .
\end{aligned}
$$

For realistic, finite in-medium path length, both effects combine in the specific interference pattern (A3).

\section{Numerical results}

In this section, we compare numerical results for the double-differential gluon distribution (3.10), its massless limit (3.11) and the dead cone approximation (3.12). We also study the transverse momentum integrated energy distribution and the energy integrated average medium-induced energy loss.

Explored parameter space. The double differential gluon distribution (3.10) for massless quarks depends on two parameters only, the characteristic gluon energy $\bar{\omega}_{c}$ and the typical transverse momentum (Debye screening mass) $\mu$. Presenting our results in rescaled variables $\bar{\kappa}^{2}=\mathbf{k}_{\perp}^{2} / \mu^{2}$ and $\bar{\gamma}=\bar{\omega}_{c} / \omega$, we explore the unrestricted parameter range for $\bar{\omega}_{c}$ and $\mu^{2}$. For the transverse momentum integrated gluon distribution (3.16), the parameter $\bar{R}=\bar{\omega}_{c} L$ enters via the integration boundary. We motivate our choice of the value of $\bar{R}$ from a model analysis of high- $p_{\perp}$ suppressed hadroproduction in central Au-Au collisions at the RHIC. The order of magnitude of the suppression is in qualitative agreement with the parameter choice $\bar{R}=2000, \bar{\omega}_{c}=67.5 \mathrm{GeV}$, and $n_{0} L=1$ (see Ref. [23]). We thus choose $\bar{R}=1000$ and a significantly larger value $\bar{R}=40000$ for numerical calculations. The latter can be viewed as an upper estimate for the LHC. If the quark mass is finite, the double differential gluon distribution also depends on the effective mass (3.9) which is a function of the ratio $m / E$ and of $\bar{R}$. We prefer to present all results in terms of $m / E$ and $\bar{R}$ although they appear in Eq. (3.10) only in one combination.

\section{1. $k_{\perp}$-differential spectrum}

As seen in Fig. 2, the transverse momentum dependence of the medium-induced gluon distribution deviates qualitatively from the dead cone approximation (3.12). First, at small transverse momentum, the medium induced gluon radiation does not vanish as for the dead cone approximation (3.12). Instead, it is enhanced even if compared to the mass-
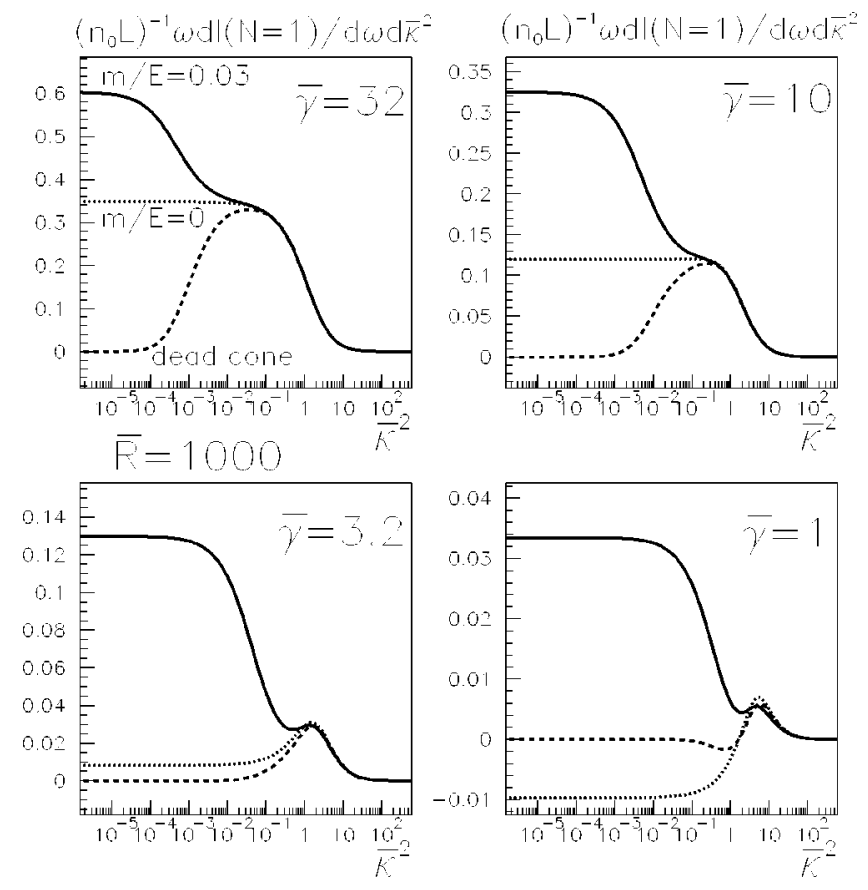

FIG. 2. The medium-induced gluon distribution as a function of the transverse momentum $\bar{\kappa}^{2}=\mathbf{k}_{\perp}^{2} / \mu^{2}$ and for different values of $\bar{\gamma}=\bar{\omega}_{c} / \omega$. Different curves correspond to the full medium-induced gluon distribution (3.10) for a mass to energy ratio 0.03 of the heavy quark (solid line), the massless limit (3.11) of this spectrum (dotted line), and its dead cone approximation (3.12) (dashed line).

less case. Second, compared to the massless case there is a suppression of the radiated gluon distribution (3.10) for intermediate and large values of $\bar{\kappa}^{2}$. This suppression is stronger for $\omega \gtrless \bar{\omega}_{c}$ (and for larger quark mass values, as can be seen in Fig. 3).

Some qualitative aspects of Fig. 2 can be viewed as resulting from the interplay of the additional radiation produced in independent medium-induced scatterings, and the coherence effects which-compared to the incoherent limit-reduce this radiation. In particular, the growth of the medium-induced gluon radiation at small transverse momentum is due to coherence effects if the coherence length is larger than the in-medium path length. For a massless quark, $\bar{l}_{\text {coh }} \sim 2 \omega / \mathbf{k}_{\perp}^{2}$ which regulates the radiation in the region $\bar{\kappa}^{2}$ $<\omega / \bar{\omega}_{c}$. This is at the origin of the plateau seen in Fig. 2 at small values of $\bar{\kappa}^{2}$. In the massive case, the phase accumulated due to scattering of the $q g$ Fock state is shifted with respect to Eq. (3.6) by $x^{2} m^{2}$ :

$$
\frac{\mathbf{k}_{\perp}^{2}}{2 \omega} L \rightarrow \frac{\mathbf{k}_{\perp}^{2}+x^{2} m^{2}}{2 \omega} L=\frac{\bar{\kappa}^{2}+\frac{1}{2}(m / E)^{2} \bar{R} / \bar{\gamma}^{2}}{\omega / \bar{\omega}_{c}} .
$$

In this case, the coherence length is smaller for the same $\mathbf{k}_{\perp}^{2}$. This shifts the coherence pattern to smaller values of the transverse momentum.

In order to understand qualitatively the filling of the dead cone at small $\bar{\kappa}^{2}$, we recall that in the limit in which a proba- 

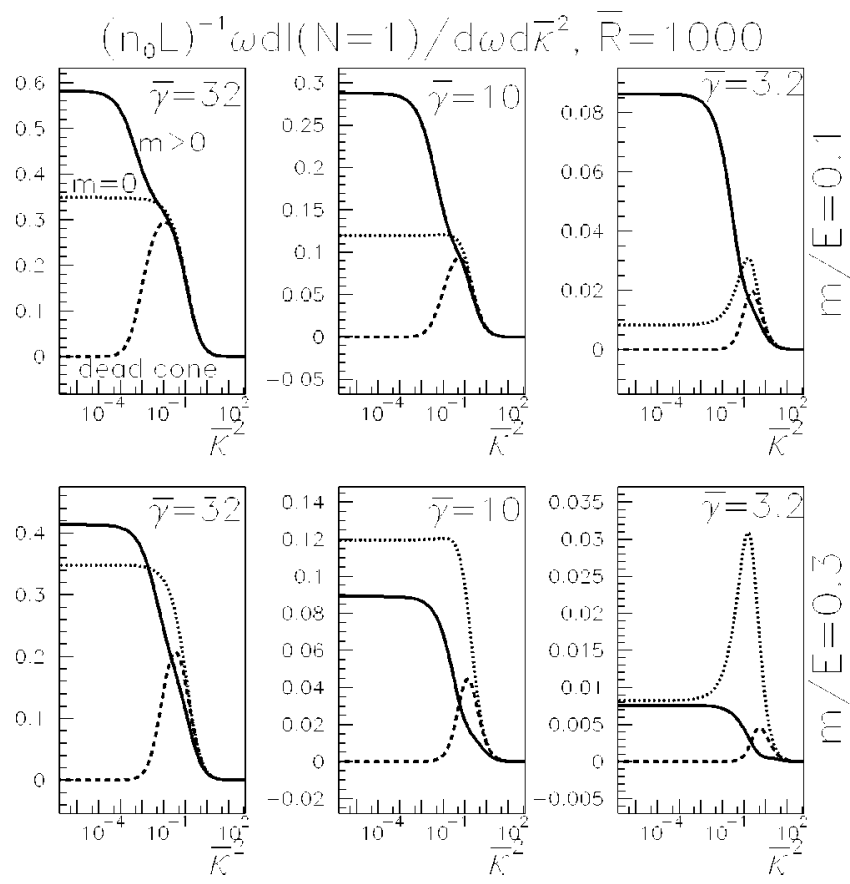

FIG. 3. Same as Fig. 2 but for the larger mass to energy ratios $m / E=0.1$ and $m / E=0.3$.

bilistic picture applies, medium modifications of the gluon radiation are determined by two competing effects [see the discussion following Eq. (3.13) and Ref. [20]]: First, additional medium-induced gluon radiation increases the gluon distribution. Second, medium-induced elastic scattering shifts emitted gluons to larger transverse momentum and thus depletes the vacuum distribution $\propto 1 / k_{\perp}^{2}$ at small transverse momentum. (For very energetic gluons which are emitted predominantly at small angle, the second mechanism dominates for massless quarks and the medium-induced part of the gluon distribution is hence negative for small transverse momentum. This is seen in the plot for $\bar{\gamma}=1$ in Fig. 2.) For massive quarks, the dead cone effect implies that there is no vacuum distribution at small angle which can be depleted due to elastic scattering. As a consequence, for massive quarks the second mechanism does not apply at small $\bar{\kappa}$ and the gluon radiation is further enhanced.

For $\bar{\kappa}^{2}>\max \left[\bar{\gamma}, \bar{M}^{2}\right]$, we expect from Eq. (3.15) that coherence effects become negligible. In the incoherent limit, Eqs. (3.13) and (3.14) indicate that mass effects reduce the medium-induced gluon radiation. This explains the depletion of the massive gluon radiation if compared to the massless limit at intermediate and large values of $\bar{\kappa}^{2}$ (see Fig. 2). For massive quarks, neither the vacuum contribution nor the medium-induced contribution to Eq. (2.4) contain collinear singularities which could enhance the importance of the region $\bar{\kappa}^{2} \ll 1$. As a consequence, parton energy loss off hard quarks will be dominated by radiation in the region $\bar{\kappa}^{2} \gtrsim 1$. There, the medium-induced radiation off massive quarks (3.10) is suppressed compared to the massless limit if the $m / E$ ratio is sufficiently large (see Fig. 3).

We have varied the mass to energy ratio $m / E$ and the parameter $\bar{R}$ in our calculation over a wide parameter range $(0.001<m / E<0.3$ and $1000<\bar{R}<40000)$. Quantitatively, the medium-induced radiation varies significantly with these parameters. Qualitatively, the effects discussed above are generic for the entire parameter space: For transverse momentum $\bar{\kappa}<\bar{M}$, medium-induced gluon radiation fills the dead cone. For a significant part of the parameter space, it is enhanced compared to the massless case, too. For very large mass to energy ratios, however, mass effects limit significantly the medium-induced gluon radiation and the radiation from massless quarks finally dominates for $\bar{\kappa}<\bar{M}$ (see Fig. $3)$.

\section{2. $k_{\perp}$-integrated spectrum}

The medium-induced gluon energy distribution obtained by integrating Eqs. (3.10), (3.11) and (3.12) over transverse momentum up to the kinematic boundary $\left|\mathbf{k}_{\perp}\right|=\omega$. In terms of the integration variable $\bar{\kappa}^{2}$, this corresponds to

$$
\omega \frac{d I(N=1)}{d \omega}=\int_{0}^{\bar{R} / 2 \bar{\gamma}^{2}} d \bar{\kappa}^{2} \omega \frac{d I(N=1)}{d \omega d \bar{\kappa}^{2}},
$$

where the integration boundary is written in terms of $\bar{R}$ $=\bar{\omega}_{c} L$.

As the phase space of the dead cone region $\bar{\kappa}^{2}<\bar{M}^{2}$ is small, the depletion of radiation off massive quarks at large $\bar{\kappa}^{2}$ compared to the massless case dominates the transverse momentum integrated gluon energy distribution (3.16) (see Fig. 4). This figure also indicates that the mass-dependent suppression of Eq. (3.16) can be even stronger than predicted by the dead cone approximation (3.12).

One may ask whether the correct amount of massdependent suppression can be estimated from a $\bar{\kappa}$-independent suppression factor $F_{\text {avdc }}(\bar{M})$ multiplying the gluon energy distribution for massless quarks. (Here, the subscript "avdc" stands for "average dead cone.") Such a factor could be useful for simplified calculations in which $\mathbf{k}_{\perp}$-dependent information is not available. Paralleling an estimate given in Ref. [25] for the dipole approximation [see Eq. (4.11) below], we have estimated $F_{\text {avdc }}(\bar{M})$ by evaluating the dead cone factor (3.7) for the characteristic angle under which medium-induced gluon radiation occurs, $\theta_{c}^{2}$ $=\mu^{2} / \omega^{2}=\left(\bar{\omega}_{c} / \omega\right)^{2}(1 / \bar{R})$. We find

$$
F_{\text {avdc }}(\bar{M})=\left(\frac{1}{1+\bar{M}^{2}}\right)^{2} .
$$

Substituting this average for the dead-cone factor in Eq. (3.12) and calculating the transverse momentum integrated spectrum, one tends to overestimate the mass-dependent reduction of the medium-induced radiation (see Fig. 4).

To gain a qualitative understanding of why the mass dependence affects the gluon distribution mainly in the region of large gluon energies in Fig. 4, we consider the radiation spectrum in the region $\bar{\kappa}>1$ which dominates Eq. (3.16). For 
$\left(n_{0} L\right)^{-1} a d l(t=1) / d \omega$
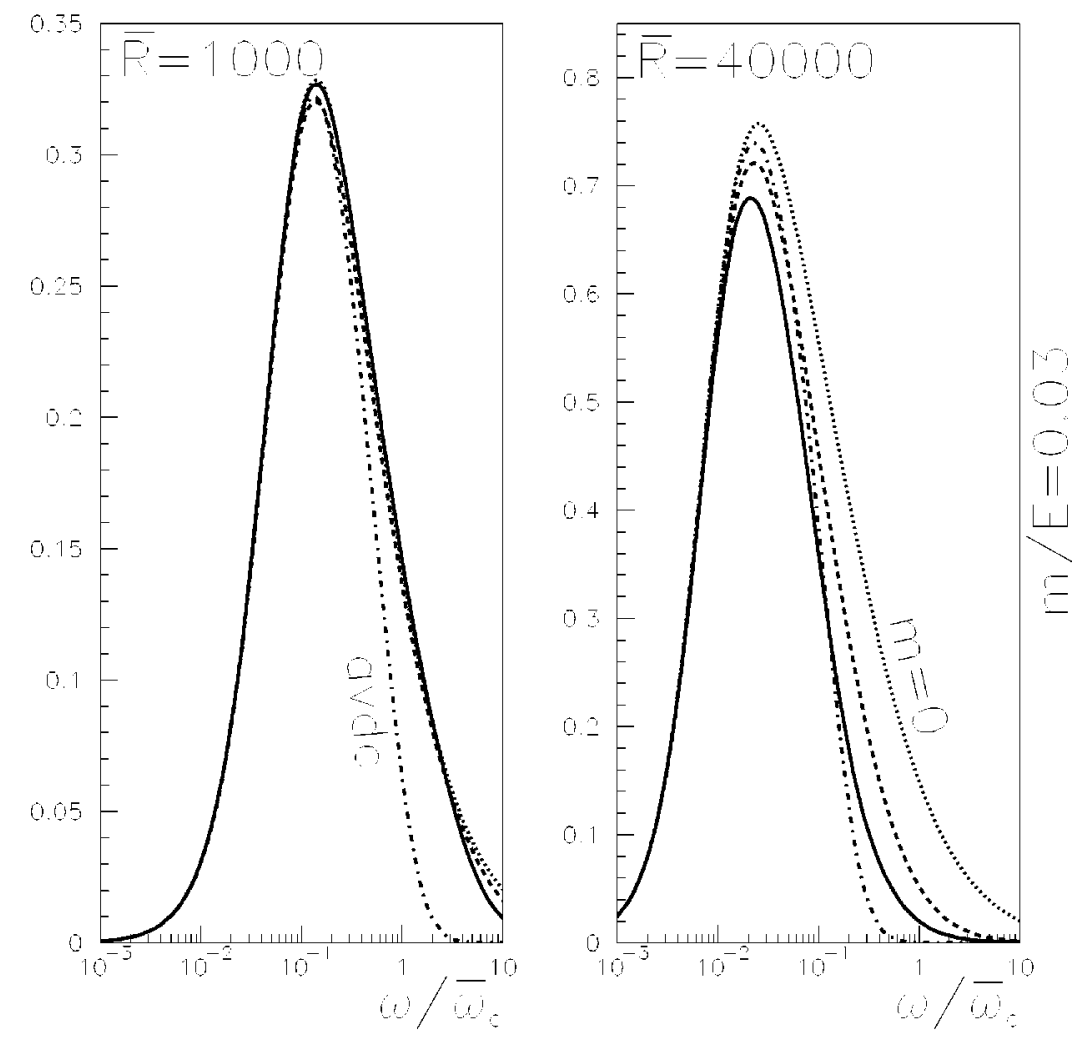

$\left.\left(n_{0} L\right)^{-1} d(1)=1\right) / d 0$

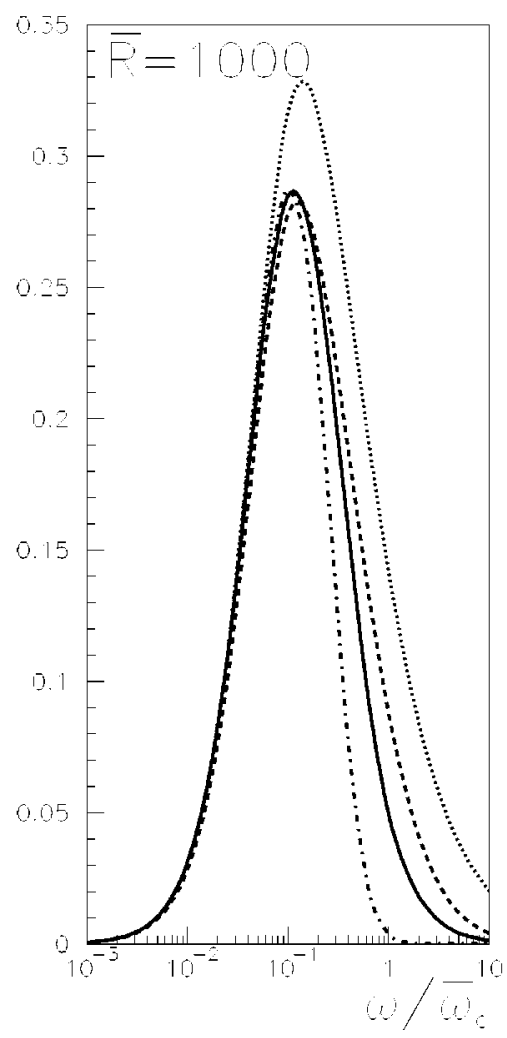

FIG. 4. The medium-induced gluon energy distribution (3.16) calculated from the full expression (3.10) for a massive quark (solid line), from the massless limit (3.11) (dotted line), from the dead-cone approximation (3.12) (dashed line) and from the average dead cone factor (3.17) multiplied by the massless spectrum (dash-dotted line). 

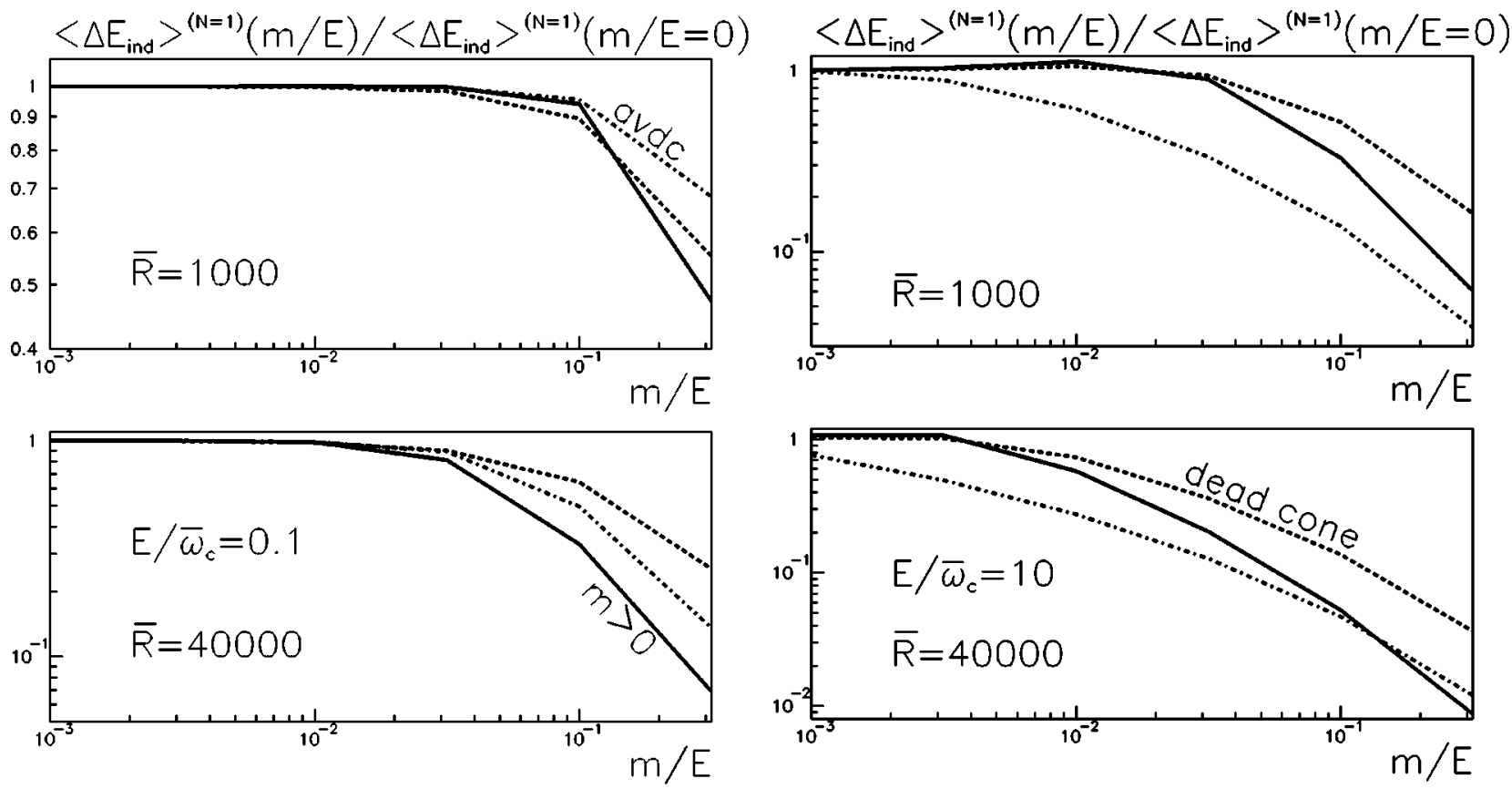

FIG. 5. The average medium-induced parton energy loss (3.21) for massive quarks, normalized to the massless limit, for different values of the density parameter $\bar{R}=\bar{\omega}_{c} L$. Curves are calculated for the full medium-induced radiation (3.10) off massive quarks (solid lines), the dead cone approximation (3.12) (dashed lines) and the corresponding expression with averaged dead cone (3.17) (dash-dotted lines).

the massless case and sufficiently large transverse momentum, the medium-induced gluon distribution shows the characteristic perturbative power law for gluon production in a hard process,

$$
\omega \frac{d I_{m=0}^{\mathrm{med}}}{d \omega d \bar{\kappa}^{2}} \propto \frac{1}{\bar{\kappa}^{4}} \quad \text { for } \quad \bar{\kappa}>1
$$

This behavior can be checked in the $N=1$ opacity expansion by taking the large- $\bar{\kappa}$ limit of Eq. (3.10). According to the condition (3.6), gluons can be emitted only for $\bar{\kappa}^{2}>\omega / \bar{\omega}_{c}$. Then,

$$
\omega \frac{d I_{m=0}^{\mathrm{med}}}{d \omega} \propto \int_{\omega / \bar{\omega}_{c}}^{\infty} \frac{d \bar{\kappa}^{2}}{\bar{\kappa}^{4}} \propto \frac{\bar{\omega}_{c}}{\omega} \text { for } \quad \omega>\bar{\omega}_{c} .
$$

This large- $\omega$ behavior is known for the $N=1$ opacity approximation $[23,38]$. In contrast to the multiple soft scattering limit studied in the next section, the region $\omega>\bar{\omega}_{c}$ dominates also the average energy loss $[23,38]$.

We now compare Eq. (3.19) to the large- $\omega$ behavior of the massive radiation spectrum. According to the dead cone approximation (3.12), mass effects deplete the distribution (3.18) for $\bar{\kappa}^{2}<\max \left[\bar{\gamma}, \bar{M}^{2}\right]$. Then, for the large- $\omega$ region where $\bar{M}^{2}>\bar{\gamma}$, the transverse momentum integrated gluon distribution can be estimated from Eq. (3.18),

$\omega \frac{d I_{m}^{\text {med }}}{d \omega} \propto \int_{\bar{M}^{2}}^{\infty} \frac{d \bar{\kappa}^{2}}{\bar{\kappa}^{4}} \propto\left(\frac{m^{2}}{E^{2}}\right) \bar{R} \frac{\bar{\omega}_{c}^{2}}{\omega^{2}}$ for $\omega>\bar{\omega}_{c} /\left(\frac{m^{2}}{E^{2}} \bar{R}\right)^{1 / 3}$.
This distribution drops off faster $\left(\propto 1 / \omega^{2}\right)$ than the corresponding massless term (3.19). We thus expect a massdependent depletion of the medium-induced gluon radiation at large gluon energy. This shows that the mass dependence reduces the medium-induced gluon radiation predominantly at large gluon energy, as seen in Fig. 4. We emphasize that the dead cone suppression is a sufficient but not a necessary condition for this large- $\omega$ behavior. What is needed is only that mass effects regulate the singularity $1 / \bar{\kappa}^{4}$ on a scale $\bar{M}$. Since the phase space for $\bar{\kappa}^{2}<\bar{M}^{2}$ is small, it is not essential whether this regulation occurs by complete extinction of the radiation e.g. via $F(\bar{\kappa}, \bar{M})$, or whether the dead cone is filled by a finite non-singular spectrum for $\bar{\kappa}^{2}<\bar{M}^{2}$. We find that the latter case is realized.

\section{Average energy loss}

The integral of Eq. (3.16) over gluon energy defines the average medium-induced energy loss

$$
\left\langle\Delta E_{\mathrm{ind}}\right\rangle=\int_{0}^{E} d \omega \omega \frac{d I(N=1)}{d \omega} .
$$

For the gluon distribution (3.10) off a massive quark, we have checked that Eq. (3.21) coincides in the $L \rightarrow \infty$ limit with the expression given by Djordjevic and Gyulassy [32]. At finite in-medium path length $L$, the differences between (3.21) and Ref. [32] may be due to the use of a different density distribution $n(\xi)$ of scattering centers.

Figure 5 shows the ratio of the medium-induced average parton energy loss (3.21) for massive quarks, normalized to the same quantity calculated for massless quarks. In general, 

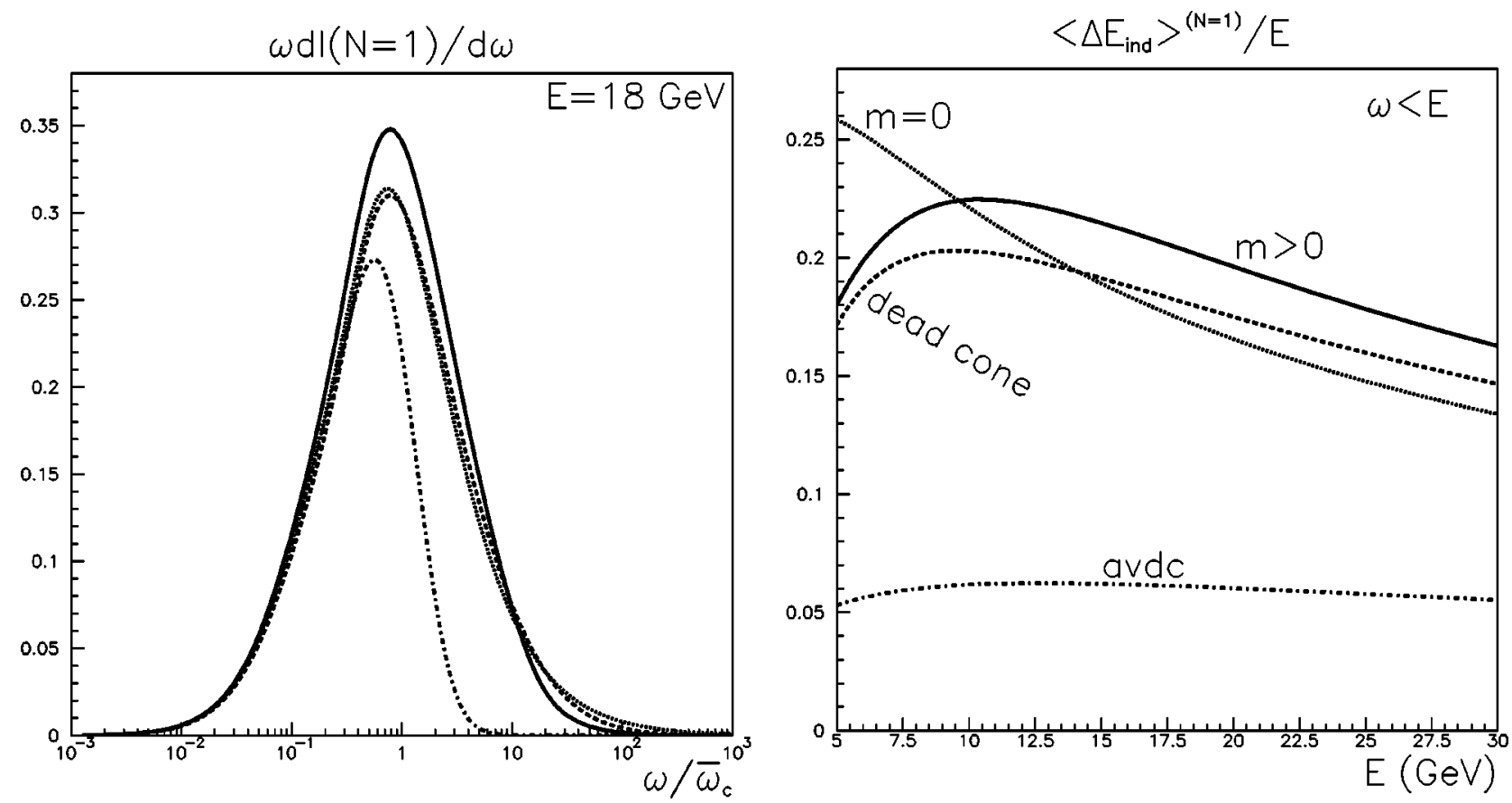

FIG. 6. For finite quark energy $E$, the normalized average energy loss (right panel) depends significantly on the kinematic boundary up to which the gluon energy distribution (left panel) is integrated. This entails significant uncertainties, which are discussed in the text. Parameter values are taken from Ref. [32].

a finite quark mass is found to reduce the parton energy loss and this reduction increases strongly with $m / E$.

We now discuss the theoretical uncertainties entering Eq. (3.21) and Fig. 5. Since the double differential distribution (3.10) is calculated in the eikonal approximation, it can have support for gluon energies $\omega>E$. This just indicates the limited validity of the eikonal approximation for small energies $E$. This issue is critical for calculations of the average parton energy loss (3.21) which can depend strongly on the phase space limit $\omega / \bar{\omega}_{c}<E / \bar{\omega}_{c}$ imposed. In Fig. 6, we illustrate this point by showing the gluon energy distribution and the average energy loss (3.21) for the parameter set $n_{0} L=4, \mu$ $=500 \mathrm{MeV}, L=4 \mathrm{fm}$ and $\alpha_{s}=0.3$. This parameter set was used to reproduce the suppression of high- $p_{\perp}$ hadroproduction observed in $\mathrm{Au}+\mathrm{Au}$ collisions at the RHIC [39], and it is used in the numerical calculation of Ref. [32] together with a charm quark mass of $m=1.5 \mathrm{GeV}$. For the standard upper integration bound $\omega<E$, the massive case agrees qualitatively in magnitude and energy dependence with the calculation shown in Fig. 1 of Ref. [32]. Small quantitative differences are due to the different finite $L$ dependence of the density distributions, as argued following Eq. (3.21). However, as seen in Fig. 6, the average energy loss (3.21) off massless quarks turns out to be smaller since a larger part of the gluon energy distribution lies above the kinematic cut. We have made no attempt to remedy this possibly unphysical behavior. We simply emphasize that any a posteriori modification of the large- $\omega$ tail of $\omega d I / d \omega$ entails significant theoretical uncertainties. It is an open problem of obvious importance to include finite energy constraints on the level of the radiation spectrum (i.e. on the level of multiple scattering Feynman diagrams) rather than on the level of the integrated parton energy loss.

\section{DIPOLE APPROXIMATION (MULTIPLE SOFT SCATTERING)}

If the medium provides a large number of soft momentum transfers, rather than a few harder ones, then the projectile performs a Brownian motion in transverse momentum. This case can be studied in the saddle point approximation of the path integral (2.1), using [34,37]

$$
n(\xi) \sigma(\mathbf{r}) \simeq \frac{1}{2} \hat{q}(\xi) \mathbf{r}^{2}
$$

Here, $\hat{q}(\xi)$ is the transport coefficient [8] which characterizes the medium-induced transverse momentum squared $\left\langle q_{\perp}^{2}\right\rangle$ transferred to the projectile per unit path length $\lambda$. In the approximation (4.1), the path integral in (2.1) is equivalent to that of a harmonic oscillator which allows for an explicit calculation.

\section{A. The vacuum term shows the dead cone effect}

For the transport coefficient $\hat{q}(\xi)=\hat{q} \Theta(L-\xi)$ of a static medium of length $L$, we evaluate the gluon distribution (2.1) by splitting the longitudinal integrals into three parts [35],

$$
\begin{aligned}
\omega \frac{d I}{d \omega d \kappa^{2}}= & \omega \frac{d I_{4}}{d \omega d \kappa^{2}}+\omega \frac{d I_{5}}{d \omega d \kappa^{2}}+\omega \frac{d I_{6}}{d \omega d \kappa^{2}} \\
= & \int_{0}^{L} d y_{l} \int_{y_{l}}^{L} d \bar{y}_{l} \ldots+\int_{0}^{L} d y_{l} \int_{L}^{\infty} d \bar{y}_{l} \ldots \\
& +\int_{L}^{\infty} d y_{l} \int_{y_{l}}^{\infty} d \bar{y}_{l} \ldots
\end{aligned}
$$


In complete analogy to the calculation for the massless case [20], one can show that the term $I_{6}$ does not depend on the medium and takes the form

$$
\omega \frac{d I_{6}}{d \omega d \mathbf{k}_{\perp}}=\frac{\alpha_{s} C_{F}}{\pi^{2}} \frac{\mathbf{k}_{\perp}^{2}}{\left(\mathbf{k}_{\perp}^{2}+x^{2} m^{2}\right)^{2}} .
$$

This expression coincides with the vacuum term (3.1). In the multiple soft scattering separation, there is hence a simple separation of the gluon distribution (2.4) into the vacuum term (4.3) and the medium-induced contribution $I_{4}+I_{5}$. The latter vanish in the absence of a medium, $\hat{q}=0$.

\section{B. Medium-induced gluon radiation}

We now discuss the medium-induced part of the gluon distribution (2.4) which in the multiple soft scattering (mss) approximation takes the form

$$
\omega \frac{d I^{\mathrm{mss}}}{d \omega d \kappa^{2}}=\omega \frac{d I_{4}}{d \omega d \kappa^{2}}+\omega \frac{d I_{5}}{d \omega d \kappa^{2}} .
$$

\section{Notation}

Paralleling the discussion of Sec. III B 1, we introduce dimensionless kinematic variables in terms of the transport coefficient $\hat{q}$ and the in-medium path length $L$. The characteristic gluon energy $\omega_{c}$, rescaled transverse momentum $\kappa$ and rescaled quark mass $M$ are defined as

$$
\begin{aligned}
\omega_{c} & =\frac{1}{2} \hat{q} L^{2}, \\
\kappa^{2} & =\frac{\mathbf{k}_{\perp}^{2}}{\hat{q} L}, \\
M^{2} & =\frac{x^{2} m^{2}}{\hat{q} L}=\frac{1}{2}\left(\frac{m^{2}}{E^{2}}\right) R \frac{\omega^{2}}{\omega_{c}^{2}}, \\
R & =\omega_{c} L .
\end{aligned}
$$

Similar to the discussion in Sec. III B 1, this defines the phase accumulated by the gluon due to multiple scattering [40]

$$
\gamma=\left\langle\frac{k_{\perp}^{2}}{2 \omega} \Delta z\right\rangle \sim \frac{\hat{q} L}{2 \omega} L=\frac{\omega_{c}}{\omega},
$$

and it allows us to write the dead cone factor as

$$
F(\kappa, M)=\left(\frac{\kappa^{2}}{\kappa^{2}+M^{2}}\right)^{2}=\left(\frac{\mathbf{k}_{\perp}^{2}}{\mathbf{k}_{\perp}^{2}+x^{2} m^{2}}\right)^{2} .
$$

\section{Analytical expressions}

In the dipole approximation, the gluon distribution (4.4) radiated off massive quarks is written in terms of the factors $I_{4}$ and $I_{5}$ which are defined in Eq. (4.2) and given explicitly
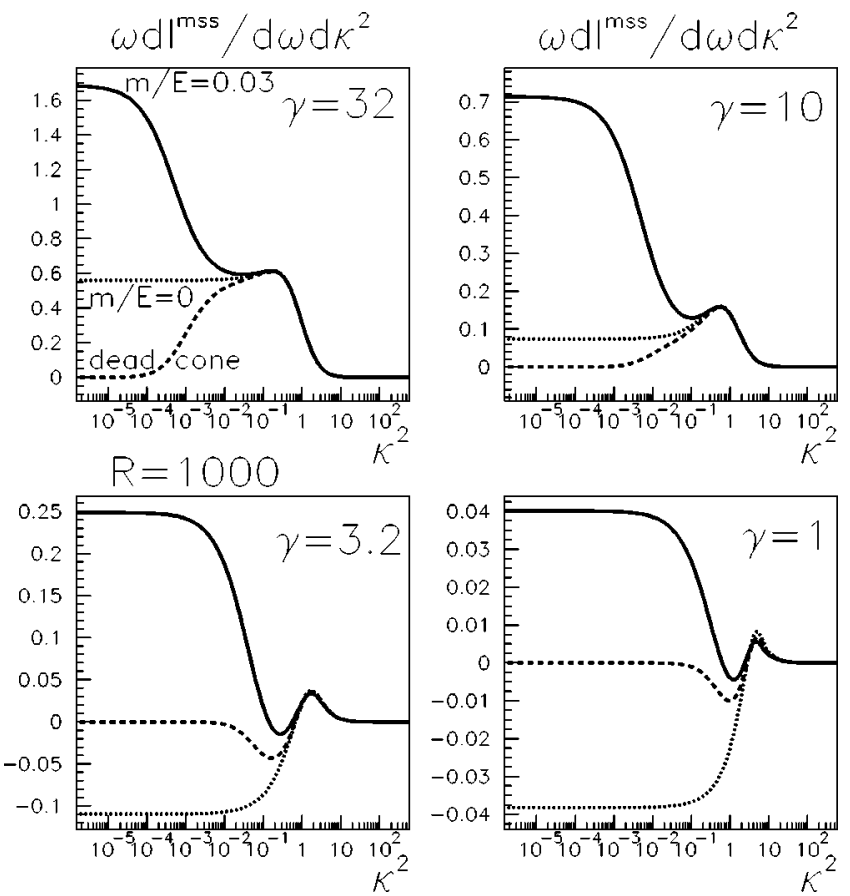

FIG. 7. The medium-induced gluon energy distribution as a function of the transverse momentum $\kappa^{2}=\mathbf{k}_{\perp}^{2} / \hat{q} L$ and for different values of $\gamma=\omega_{c} / \omega$, calculated in the multiple soft scattering approximation. Different curves correspond to the full mediuminduced gluon distribution for a mass to energy ratio 0.03 of the heavy quark (solid line), the massless limit of this spectrum (dotted line), and its dead cone approximation (4.10) (dashed line).

in Appendix B. In the following, we compare the gluon distribution (4.4) to the massless limit and to the dead cone approximation

$$
\omega \frac{d I_{\mathrm{dead}}^{\mathrm{mss}}}{d \omega d \kappa^{2}}=F(\kappa, M) \omega \frac{d I_{m=0}^{\mathrm{mss}}}{d \omega d \kappa^{2}} .
$$

\section{Numerical results}

Explored parameter space. In analogy to the study in Sec. III C, we present all results in rescaled variables $\kappa^{2}$ and $\gamma$. The parameter $R=\omega_{c} L$ is explored for the values 1000 and 40000. See Sec. III C for further details.

\section{1. $k_{\perp}$-differential spectrum}

We have calculated the transverse momentum dependence of the medium-induced gluon distribution (4.4) in the multiple soft scattering approximation. As seen in Fig. 7, the results are in qualitative agreement with those obtained in the opacity expansion (see Fig. 4 and Sec. III C for discussion). In particular, we find that the dead cone is filled by mediuminduced gluon radiation and that this radiation is depleted at large $\kappa^{2}$ compared to the massless case. The first feature persists and the second becomes more evident when going to larger mass to energy ratios (data not shown) in qualitative agreement with Fig. 3.

A qualitative understanding of these features can be based on the typical phase accumulated by the $q g$ Fock state: De- 
structive interference reduces gluon radiation if $\left(\mathbf{k}_{\perp}^{2}\right.$ $\left.+x^{2} m^{2}\right) / 2 \omega=\left(\kappa^{2}+M^{2}\right) \gamma<1$. This regulates the radiation for small $\kappa^{2}$. With increasing mass, destructive interference occurs for smaller values of $\kappa^{2}$. This explains why in comparison to the massless limit, the small- $\kappa^{2}$ plateau of the radiation spectrum in Fig. 7 is reached for smaller values of $\kappa^{2}$ in the massive case. For large $\kappa^{2}$, on the other hand, the incoherent limit is approached, where the mass dependence suppresses incoherent gluon production. Again, this is in qualitative agreement with the mass dependence seen in Fig. 7.

\section{2. $k_{\perp}$-integrated spectrum}

The transverse momentum integrated gluon energy distribution calculated from Eq. (4.4) is shown in Fig. 8. This integral is dominated by the phase space region $\kappa^{2}>M^{2}$, and thus does not depend strongly on the finite medium-induced radiation inside the dead cone region. Compared to the massless case, the large- $\omega$ tail of the gluon energy distribution is depleted with increasing $m / E$ ratio. The approximation of this effect by the dead cone (4.10) tends to underestimate this depletion. These findings are in qualitative agreement with the results reported for the opacity expansion in Fig. 4. For a qualitative understanding of why the mass dependence predominantly depletes the gluon radiation at large $\omega$, one can recall again that the mass-dependent suppression of smallangle radiation predominantly depletes the hard part of the spectrum.

We have also tested numerically the approximation of Dokshitzer and Kharzeev [25]. These authors replaced the transverse momentum dependent dead cone factor in Eq. (4.10) by an average expression, evaluated at the characteristic angle $\theta_{c}^{2} \simeq \sqrt{2} \gamma^{3 / 2} / R$ under which medium-induced gluon radiation occurs on average,

$$
F_{\mathrm{DK}}=\left(\frac{1}{1+1 / \sqrt{2} m^{2} / E^{2} R / \gamma^{3 / 2}}\right)^{2} .
$$

This approximation allows us to mimic the qualitative trend but is found to overestimate the depletion of the large- $\omega$ tail significantly (see Fig. 8).

\section{Average energy loss}

From the gluon energy distribution, we have calculated the average parton energy loss according to Eq. (3.21). As seen in Fig. 9, a finite quark mass reduces parton energy loss significantly for sufficiently large mass to energy ratios, $m / E>0.1$ say. For smaller mass to energy ratios, there is some parameter range where our numerical results indicate the opposite effect. However, similar to the case of the opacity expansion, this may be an artifact of the phase space constraint in the definition of the average energy loss (3.21) (see the discussion of Figs. 5 and 6 above). To explore this theoretical uncertainty, we have paralleled the logic of Sec. III. We have calculated in Fig. 10 the gluon energy distribution (4.4) for the set of parameters which reproduce the nuclear modification factor for central $\mathrm{Au}+\mathrm{Au}$ collisions at the RHIC [23]. Integrating this gluon energy distribution for
$\omega<E$, we find again that the average parton energy loss for massless quarks can be larger than that for massive ones, simply because the kinematic boundary $\omega<E$ cuts off the more pronounced large $\omega$-tail of the gluon energy distribution for $m=0$. For the reasons given in Sec. III C, we conclude that this motivates us to go beyond the high-energy approximation and to include finite energy constraints in the calculation of $\omega d I / d \omega$ rather than to impose them a posteriori in the integral over $\omega d I / d \omega$.

\section{CONCLUSION}

Early studies demonstrated that the transverse momentum spectra of charmed and beauty hadrons in nucleus-nucleus collisions depend strongly on the assumed parton energy loss [41-43]. More recently, the quark mass dependence of this effect was argued to give access to the detailed mechanism of medium-modified parton fragmentation [25,30-33]. To better assess this mechanism, we have presented here the first study of the transverse momentum and energy dependence of the medium-induced gluon radiation off massive quarks.

Our calculation is based on the path-integral formalism (2.1) which resums medium modifications to leading order in $1 / E$ and to all orders in opacity. We have employed two approximation schemes which model the medium dependence of the hard parton fragmentation as a series of many soft gluon exchanges or as a single semi-hard momentum transfer. Despite these different physical pictures, both approaches lead to comparable results, in agreement with an earlier study of the massless case [23].

We find that medium-induced gluon radiation generically fills the dead cone $\theta<m / E$ (see Figs. 2, 3 and 7). However, in comparison to the transverse momentum integrated gluon energy distribution radiated off massless quarks, the radiation is depleted at large gluon energies (see Figs. 4 and 8). The average parton energy loss results from an interplay of both effects and tends to be smaller for massive quarks than for massless ones (see Figs. 5 and 9). However, for sufficiently small parton energies $E$, the kinematic boundary $\omega$ $<E$ can lead to the peculiar case that the average parton energy loss is larger for massive quarks than for massless ones (see Figs. 6 and 10). We have argued that this behavior may be unphysical and limits the application of the formalism of Refs. [8-12] to sufficiently large quark energies; see the discussion at the end of Sec. III C.

As of today, the only experimental information about open charm production in $\mathrm{Au}+\mathrm{Au}$ collisions at the RHIC is the prompt electron spectrum measured by PHENIX at $\sqrt{s_{\mathrm{NN}}}=130 \mathrm{GeV}$ [44]. It was argued that these data do not indicate a significant parton energy loss for charm quarks. But it is equally true that they do not constrain parton energy loss significantly. First, the experimental errors on the prompt electron spectrum are still large. Second, for the measured transverse momentum range $p_{\perp}^{\text {electron }}<3 \mathrm{GeV}$, the correlation between the transverse momentum of the electron and of the charmed hadron is very weak. Both complicate any conclusion about the medium-induced degradation of $p_{\perp}^{\text {charm }}$. In addition, the moderate values of $p_{\perp}^{\text {charm }}$ accessed by PHENIX correspond to charm quarks which move rather 
$\omega \mathrm{dl}^{\mathrm{mss}} / \mathrm{d} \omega$
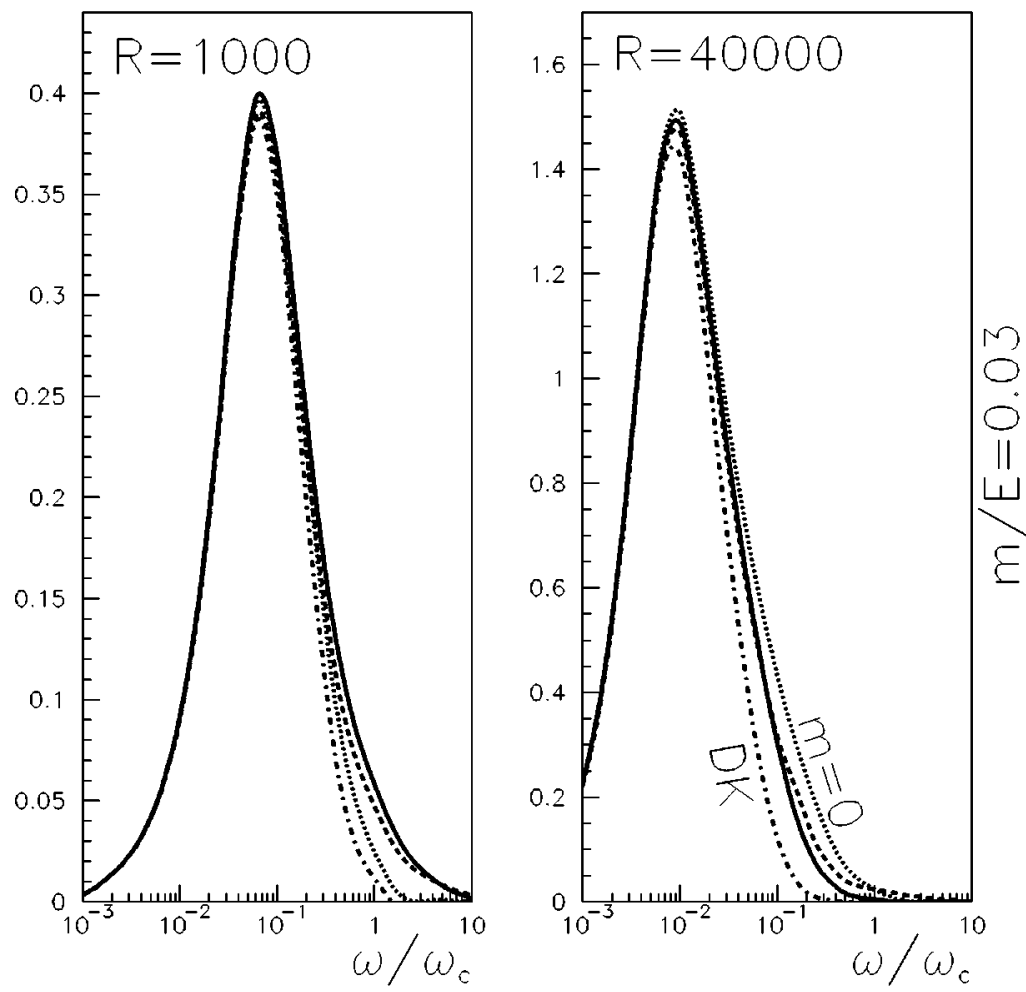

$$
\omega \mathrm{dl}^{\mathrm{mss}} / \mathrm{d} \omega
$$

FIG. 8. Same as Fig. 4, here calculated in the multiple soft scattering approximation.
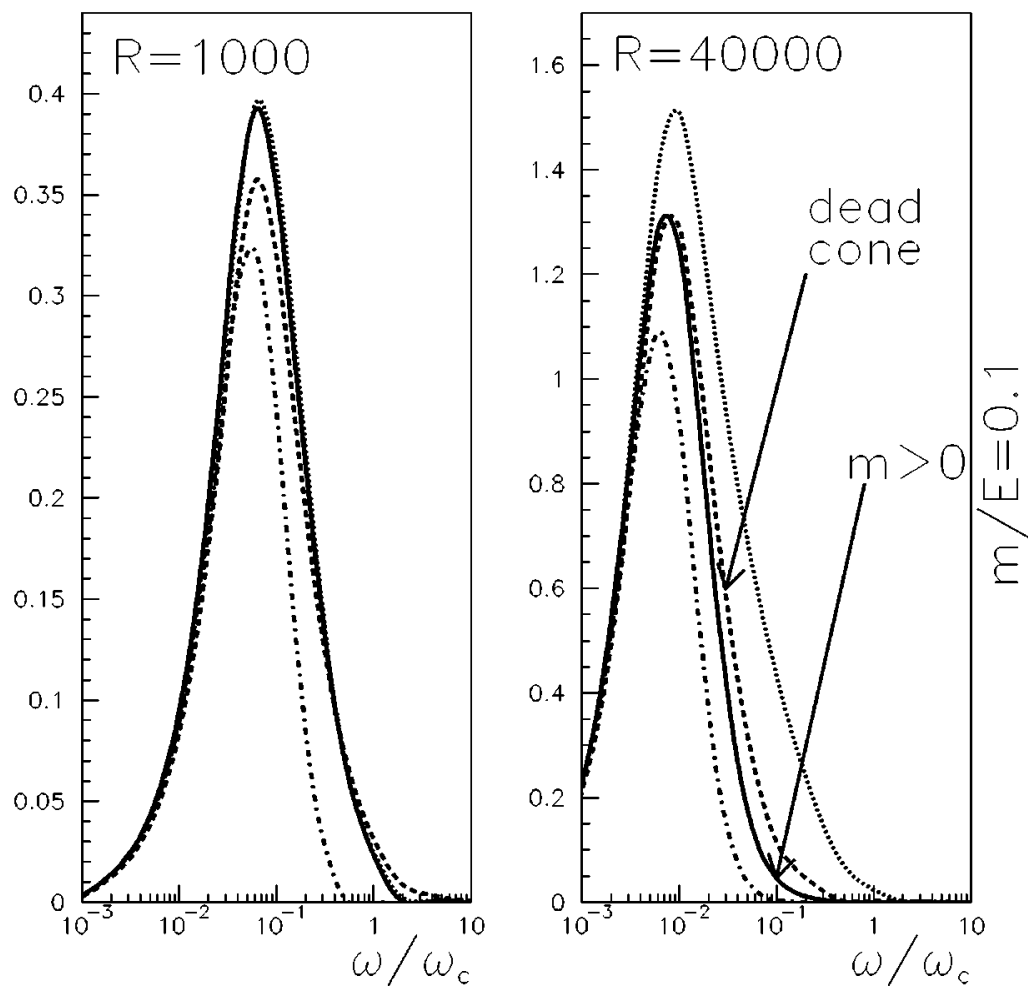

slowly and thus may turn into hadrons prior to leaving the collision region. In this case, the energy degradation of charmed hadrons would get contributions from their hadronic cross sections and the formalism employed here has to be revisited.

The experimental information from RHIC is expected to improve soon. First, the higher statistics of future runs will allow measurement of the prompt electron spectrum in a 

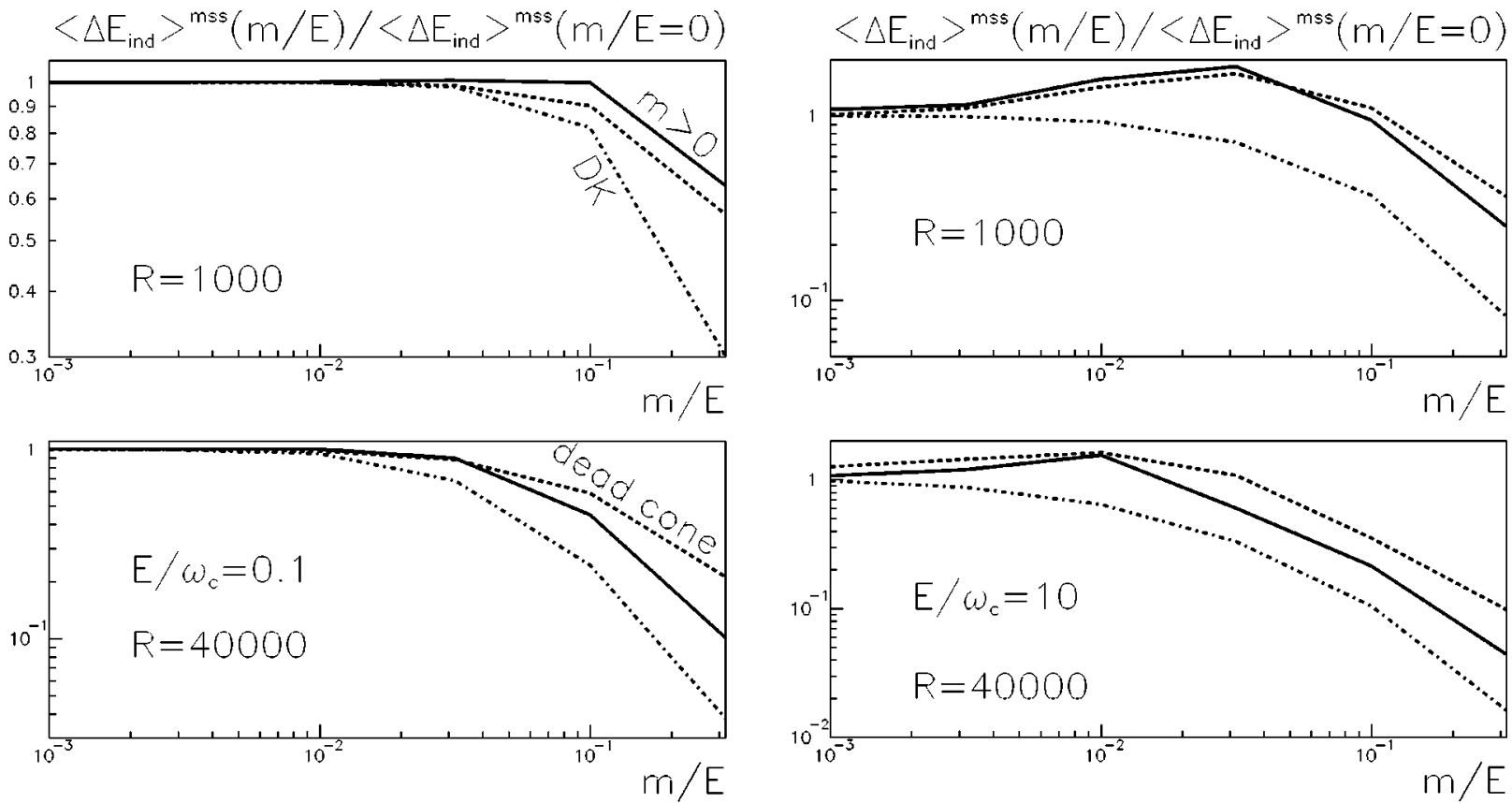

FIG. 9. Same as Fig. 5, here calculated in the multiple soft scattering approximation.

wider $p_{\perp}$ range. Second, the topological reconstruction of the hadronic decay of charmed hadrons (via $D^{0} \rightarrow K^{-} \pi^{+}$and several three-body decays), already performed in $d+\mathrm{Au}$ [45], should provide a more direct measurement out to significant transverse momentum. On a longer time scale, open charm measurements at the LHC will further extend this $p_{\perp}$ range to $p_{\perp}^{D^{0}} \sim 15 \mathrm{GeV}$ [46]. Also, the energy loss of $b$-flavored quarks is expected to become accessible at the LHC via high-mass dimuon and secondary $\mathrm{J} / \psi$ production [47]. Despite the significant uncertainties of our calculation discussed above, there is one conclusion which we can draw: parton energy loss is reduced by mass effects, but for realistic parameter values it remains sizable. Thus, our study favors a medium-induced enhancement of the $D^{0} / \pi^{0}$ ratio at
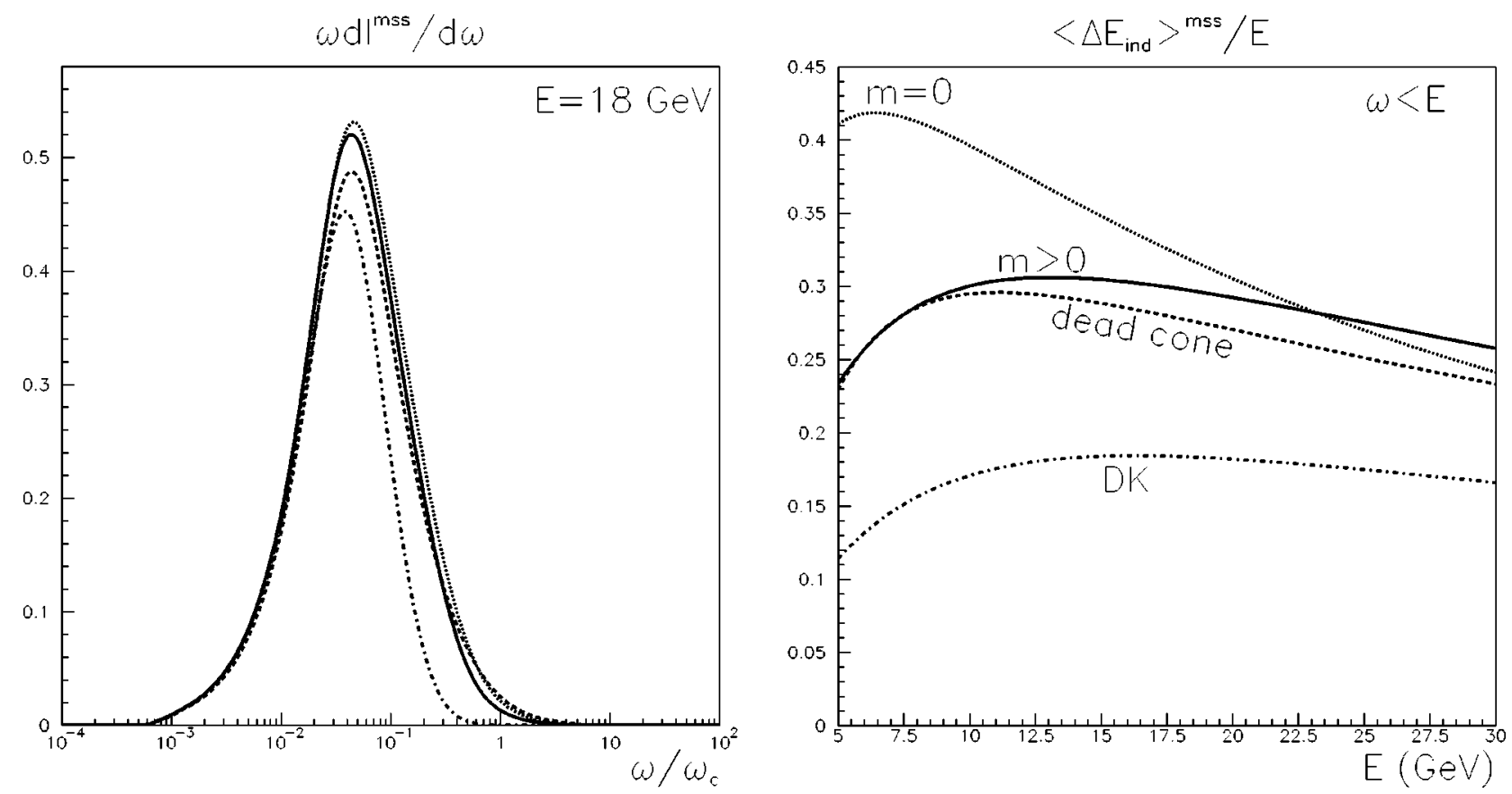

FIG. 10. Same as Fig. 6, here calculated in the multiple soft scattering approximation for parameter values $R=2000, \omega_{c}=67.5 \mathrm{GeV}$ and $m=1.5 \mathrm{GeV}$. This figure allows us to illustrate the uncertainties in calculations of the average parton energy loss for finite energy quarks (see text). 
sufficiently large transverse energy but we still expect the nuclear modification factor for $D^{0}$ to lie below unity.

\section{ACKNOWLEDGMENTS}

We thank R. Baier, A. Dainese, K. Eskola, H. Honkanen, A. Morsch, G. Rodrigo, and J. Schukraft for helpful discussions.

\section{APPENDIX A: GLUON ENERGY DISTRIBUTION TO FIRST ORDER IN OPACITY}

In this appendix, we give details for the calculation of the zeroth and first order in opacity of the gluon energy distribution (2.1). We start by expanding the path integral in Eq. (2.1) in powers of $n(\xi) \sigma(\mathbf{r})$,

$$
\begin{aligned}
\mathcal{K}\left(\mathbf{r}, y_{l} ; \overline{\mathbf{r}}, \bar{y}_{l}\right)= & \mathcal{K}_{0}\left(\mathbf{r}, y_{l} ; \overline{\mathbf{r}}, \bar{y}_{l}\right)-\int_{z}^{z^{\prime}} d \xi n(\xi) \int d \boldsymbol{\rho} \mathcal{K}_{0}\left(\mathbf{r}, y_{l} ; \boldsymbol{\rho}, \xi\right) \frac{1}{2} \sigma(\boldsymbol{\rho}) \mathcal{K}_{0}\left(\boldsymbol{\rho}, \xi ; \overline{\mathbf{r}}, \bar{y}_{l}\right) \\
& +\int_{z}^{z^{\prime}} d \xi_{1} n\left(\xi_{1}\right) \int_{\xi_{1}}^{z^{\prime}} d \xi_{2} n\left(\xi_{2}\right) \int d \boldsymbol{\rho}_{1} d \boldsymbol{\rho}_{2} \mathcal{K}_{0}\left(\mathbf{r}, y_{l} ; \boldsymbol{\rho}_{1}, \xi_{1}\right) \frac{1}{2} \sigma\left(\boldsymbol{\rho}_{1}\right) \mathcal{K}\left(\boldsymbol{\rho}_{1}, \xi_{1} ; \boldsymbol{\rho}_{2}, \xi_{2}\right) \frac{1}{2} \sigma\left(\boldsymbol{\rho}_{2}\right) \mathcal{K}_{0}\left(\boldsymbol{\rho}_{2}, \xi_{2} ; \overline{\mathbf{r}}, \bar{y}_{l}\right) .
\end{aligned}
$$

Here, the free path integral $\mathcal{K}_{0}$ is of Gaussian form

$$
\mathcal{K}_{0}\left(\mathbf{r}, y_{l} ; \overline{\mathbf{r}}, \bar{y}_{l}\right)=\frac{\omega}{2 \pi i\left(\bar{y}_{l}-y_{l}\right)} \exp \left\{\frac{i \omega(\overline{\mathbf{r}}-\mathbf{r})^{2}}{2\left(\bar{y}_{l}-y_{l}\right)}\right\} .
$$

We expand the integrand of Eq. (2.1) to first order in $n(\xi) \sigma(r)$. The longitudinal integrals in Eq. (2.1) are regularized in intermediate steps of the calculation, as explained in Refs. [10,35]. We note that the $N$ th order of Eq. (2.1) involves $2 N+1$ terms only. This makes it straightforward to obtain explicit expressions to high orders in opacity.

Inserting Eq. (A1) into Eq. (2.1), one finds to zeroth order the opacity term (3.1). To first order in opacity, one finds

$$
\begin{aligned}
\omega \frac{d I(N=1)}{d \omega d \mathbf{k}_{\perp}}= & \frac{\alpha_{s} C_{F}}{\pi^{2}} 4 n_{0} \omega \int \frac{d \mathbf{q}}{(2 \pi)^{2}}|a(\mathbf{q})|^{2} \\
& \times \frac{L Q_{1}-\sin \left(L Q_{1}\right)}{\left[\left(\mathbf{k}_{\perp}+\mathbf{q}\right)^{2}+x^{2} m^{2}\right]^{2}}
\end{aligned}
$$

$$
\times\left[\frac{\left(\mathbf{k}_{\perp}+\mathbf{q}\right)^{2}}{\left(\mathbf{k}_{\perp}+\mathbf{q}\right)^{2}+x^{2} m^{2}}-\frac{\mathbf{k}_{\perp} \cdot\left(\mathbf{k}_{\perp}+\mathbf{q}\right)}{\mathbf{k}_{\perp}^{2}+x^{2} m^{2}}\right],
$$

where the transverse energy of the scattered gluon is $Q_{1}$ $=\left[\left(\mathbf{k}_{\perp}+\mathbf{q}\right)^{2}+x^{2} m^{2}\right] / 2 \omega$.

\section{$N=1$ opacity term for Yukawa-type scattering potential}

We have studied Eq. (A3) for arbitrary in-medium path length for a Yukawa-type elastic scattering center with Debye screening mass $\mu$ :

$$
|a(\mathbf{q})|^{2}=(2 \pi)^{2} \frac{\mu^{2}}{\pi\left(\mathbf{q}^{2}+\mu^{2}\right)^{2}} .
$$

Shifting in Eq. (A3) the relative momentum integration $\mathbf{q}$ $\rightarrow \mathbf{q}+\mathbf{k}$ and doing the angular integrations, one finds

$$
\omega \frac{d I(N=1)}{d \omega d k^{2}}=\frac{\alpha_{s} C_{F}}{\pi} \frac{n_{0} \mu^{2}}{\omega} \int_{0}^{\infty} d q^{2} \frac{L Q_{1}^{\prime}-\sin \left(L Q_{1}^{\prime}\right)}{Q_{1}^{\prime 2}} \frac{q^{2}}{q^{2}+x^{2} m^{2}} \frac{\mu^{2}\left(k^{2}+x^{2} m^{2}\right)+\left(k^{2}-x^{2} m^{2}\right)\left(k^{2}-q^{2}\right)}{\left(k^{2}+x^{2} m^{2}\right)\left[\left(\mu^{2}+k^{2}+q^{2}\right)^{2}-4 k^{2} q^{2}\right]^{3 / 2}},
$$

where $Q_{1}^{\prime}=\left(\mathbf{q}^{2}+x^{2} m^{2}\right) / 2 \omega$. From this expression, we find Eq. (3.10) by rescaling the variables $\omega, k^{2}$ and $m^{2}$ to the dimensionless variables $\bar{\gamma}, \bar{\kappa}^{2}$ and $\bar{M}^{2}$ defined in Eqs. (3.4), (3.7) and (3.9) respectively.

\section{APPENDIX B: GLUON ENERGY DISTRIBUTION IN THE DIPOLE APPROXIMATION}

Here, we give the full expression for the medium-induced part of the double differential gluon distribution (4.4). The 
calculation is done in complete analogy to the calculation of the massless case [20], but keeping the mass dependence of Eq. (2.1). In the rescaled dimensionless variables introduced in Sec. IV, one finds

$$
\begin{aligned}
\omega \frac{d I_{4}}{d \omega d \kappa^{2}}= & \frac{\alpha_{s} C_{F}}{\pi} \gamma^{2} 2 \operatorname{Re} \int_{0}^{1} d t \int_{t}^{1} d \bar{t} e^{i M^{2} \gamma(t-\bar{t})} \\
& \times \exp \left[-\frac{\kappa^{2}}{4\left(D_{4}-i A_{4} B_{4}\right)}\right] \\
& \times\left[\frac{i A_{4}^{3} B_{4} \kappa^{2}}{\left(D_{4}-i A_{4} B_{4}\right)^{3}}-\frac{4 A_{4}^{2} D_{4}}{\left(D_{4}-i A_{4} B_{4}\right)^{2}}\right],
\end{aligned}
$$

where

$$
\Omega=\frac{1-i}{\sqrt{2}} \sqrt{\gamma}
$$

and

$$
\begin{gathered}
A_{4}=\frac{\Omega}{4 \gamma \sin [\Omega(\bar{t}-t)]}, \quad B_{4}=\cos [\Omega(\bar{t}-t)], \\
D_{4}=\frac{1}{4}(1-\bar{t}) .
\end{gathered}
$$

The term $I_{5}$ in Eq. (4.2) takes the form

$$
\begin{aligned}
\omega \frac{d I_{5}}{d \omega d \kappa^{2}}= & \frac{\alpha_{s} C_{F}}{\pi} \gamma 2 \operatorname{Re} \int_{0}^{1} d t e^{-i M^{2} \gamma t}\left(\frac{-i \kappa^{2}}{\kappa^{2}+M^{2}}\right) \frac{1}{B_{5}^{2}} \\
& \times \exp \left[-\frac{i \kappa^{2}}{4 A_{5} B_{5}}\right],
\end{aligned}
$$

where

$$
A_{5}=\frac{\Omega}{4 \gamma \sin [\Omega t]}, \quad B_{5}=\cos [\Omega t] .
$$

[1] Y.L. Dokshitzer, V.A. Khoze, and S.I. Troian, J. Phys. G 17, 1481 (1991).

[2] OPAL Collaboration, K. Ackerstaff et al., Eur. Phys. J. C 7, 369 (1999).

[3] ALEPH Collaboration, D. Buskulic et al., Z. Phys. C 62, 1 (1994)

[4] OPAL Collaboration, G. Abbiendi et al., hep-ex/0210031.

[5] B.A. Schumm, Y.L. Dokshitzer, V.A. Khoze, and D.S. Koetke, Phys. Rev. Lett. 69, 3025 (1992).

[6] S. Gieseke, P. Stephens, and B. Webber, J. High Energy Phys. 12, 045 (2003).

[7] M. Gyulassy and X.N. Wang, Nucl. Phys. B420, 583 (1994).

[8] R. Baier, Y.L. Dokshitzer, A.H. Mueller, S. Peigne, and D. Schiff, Nucl. Phys. B484, 265 (1997).

[9] B.G. Zakharov, JETP Lett. 65, 615 (1997).

[10] U.A. Wiedemann, Nucl. Phys. B588, 303 (2000).

[11] M. Gyulassy, P. Levai, and I. Vitev, Nucl. Phys. B594, 371 (2001).

[12] X.N. Wang and X.f. Guo, Nucl. Phys. A696, 788 (2001).

[13] PHENIX Collaboration, K. Adcox et al., Phys. Rev. Lett. 88, 022301 (2002).

[14] PHENIX Collaboration, S.S. Adler, Phys. Rev. C 69, 034910 (2004).

[15] STAR Collaboration, C. Adler et al., Phys. Rev. Lett. 89, 202301 (2002).

[16] STAR Collaboration, J. Adams et al., Phys. Rev. Lett. 91, 172302 (2003).

[17] PHOBOS Collaboration, B.B. Back et al., Phys. Lett. B 578, 297 (2004).

[18] BRAHMS Collaboration, I. Arsene et al., Phys. Rev. Lett. 91, 072305 (2003).

[19] X.N. Wang, Phys. Lett. B 579, 299 (2004).

[20] U.A. Wiedemann, Nucl. Phys. A690, 731 (2001).
[21] R. Baier, Y.L. Dokshitzer, A.H. Mueller, and D. Schiff, Phys. Rev. C 60, 064902 (1999).

[22] R. Baier, Y.L. Dokshitzer, A.H. Mueller, and D. Schiff, Phys. Rev. C 64, 057902 (2001).

[23] C.A. Salgado and U.A. Wiedemann, Phys. Rev. D 68, 014008 (2003).

[24] C.A. Salgado and U.A. Wiedemann, hep-ph/0310079.

[25] Yu.L. Dokshitzer and D.E. Kharzeev, Phys. Lett. B 519, 199 (2001).

[26] K.J. Eskola, V.J. Kolhinen, and R. Vogt, Phys. Lett. B 582, 157 (2004).

[27] F. Gelis and R. Venugopalan, Phys. Rev. D 69, 014019 (2004).

[28] D. Kharzeev and K. Tuchin, Nucl. Phys. A735, 248 (2004).

[29] A. Accardi et al., hep-ph/0308248.

[30] M. Djordjevic and M. Gyulassy, Phys. Lett. B 560, 37 (2003).

[31] M. Djordjevic and M. Gyulassy, Phys. Rev. C 68, 034914 (2003).

[32] M. Djordjevic and M. Gyulassy, Nucl. Phys. A733, 265 (2004).

[33] B.W. Zhang, E. Wang, and X.N. Wang, nucl-th/0309040.

[34] B.G. Zakharov, JETP Lett. 63, 952 (1996).

[35] U.A. Wiedemann and M. Gyulassy, Nucl. Phys. B560, 345 (1999).

[36] C.A. Salgado and U.A. Wiedemann, Phys. Rev. Lett. 89, 092303 (2002).

[37] B.G. Zakharov, Yad. Fiz. 61924 (1998) [Phys. At. Nucl. 61, 838 (1998)].

[38] M. Gyulassy, P. Levai, and I. Vitev, Phys. Rev. Lett. 85, 5535 (2000).

[39] P. Levai, G. Papp, G. Fai, M. Gyulassy, G.G. Barnafoldi, I. Vitev, and Y. Zhang, Nucl. Phys. A698, 631 (2002).

[40] R. Baier, Nucl. Phys. A715, 209 (2003).

[41] E.V. Shuryak, Phys. Rev. C 55, 961 (1997).

[42] Z.w. Lin, R. Vogt, and X.N. Wang, Phys. Rev. C 57, 899 (1998). 
[43] M.G. Mustafa, D. Pal, D.K. Srivastava, and M. Thoma, Phys. Lett. B 428, 234 (1998).

[44] PHENIX Collaboration, K. Adcox et al., Phys. Rev. Lett. 88, 192303 (2002).

[45] STAR Collaboration, A. Tai, nucl-ex/0404029, talk at the XVIIth International Conference on Ultra-Relativistic
Nucleus-Nucleus Collisions (Quark Matter 2004), Oakland, 2004.

[46] ALICE Collaboration, A. Dainese, Eur. Phys. J. C 33, 495 (2004).

[47] I.P. Lokhtin and A.M. Snigirev, J. Phys. G 27, 2365 (2001). 